\title{
Effects of exogenous surfactant instillation in clinical lung transplantation: A prospective, randomized trial
}

\author{
Martin Strüber, MD, a Stefan Fischer, MD, MSc, ${ }^{a}$ Jost Niedermeyer, MD, ${ }^{\text {b }}$ Gregor Warnecke, MD, ${ }^{a}$ \\ Bernhard Gohrbandt, MD, ${ }^{a}$ Adelheid Görler, MD, ${ }^{a}$ Andre R. Simon, MD, ${ }^{a}$ Axel Haverich, MD, and Jens M. Hohlfeld, MD ${ }^{b}$
}

From the Hannover Thoracic Transplant Program, Division of Thoracic and Cardiovascular Surgery a and the Department of Respiratory Medicine, ${ }^{\mathrm{b}}$ Hannover Medical School, Hannover, Germany.

Received for publication April 11, 2006; revisions received Dec 10, 2006; accepted for publication Dec 14, 2006.

Address for reprints: Martin Strüber, MD, Director, Hannover Thoracic Transplant Program, Division of Thoracic and Cardiovascular Surgery, Hannover Medical School, Carl Neuberg Strasse 1, 30625 Hannover, Germany (E-mail: strueber.martin@mh-hannover.de).

J Thorac Cardiovasc Surg 2007;133:1620-5 $0022-5223 / \$ 32.00$

Copyright $\odot 2007$ by The American Association for Thoracic Surgery

doi:10.1016/j.jtcvs.2006.12.057
Objective: Despite the introduction of low potassium-based preservation strategies for clinical lung transplantation, relevant early graft dysfunction occurs in up to $20 \%$ of cases after lung transplantation. This was found to be frequently associated with postreperfusion surfactant dysfunction. We performed a randomized, prospective study investigating the effect of exogenous surfactant instillation into human donor lungs on posttransplant surfactant function and on clinical outcome.

Methods: Exogenous surfactant was instilled into 15 donor lungs before retrieval via bronchoscopy. Bronchoalveolar lavage fluids were taken before instillation as well as 24 hours after transplantation. Surfactant function, phospholipids, and protein content in bronchoalveolar lavage fluids were assessed and clinical data prospectively recorded. Pulmonary function testing was performed 4 weeks after lung transplantation. Additionally, the best forced expiratory volume in 1 second was determined within the first year after lung transplantation. The control group consisted of 14 patients receiving donor lungs without surfactant instillation in randomized order. Pulmonary function test results were further compared with those of 154 consecutive recipients of bilateral lung transplants, which were not involved in the study (historical control).

Results: No deaths occurred during the first year after lung transplantation. Surfactant function in donor lungs was within normal ranges before harvest. In the control group, surfactant function was markedly impaired after reperfusion. This was significantly improved by surfactant substitution. Protein content of the bronchoalveolar lavage fluid in the surfactant group was significantly lower, indicating less leakage through the alveolocapillary membrane. Forced expiratory volume in 1 second after 4 weeks was significantly higher in the surfactant group than in either control group ( $P=.034$ and .01 , respectively). Interestingly, the best forced expiratory volume in 1 second during the first year after lung transplantation was significantly higher in both control groups compared with forced expiratory volume measured in examinations 4 weeks after lung transplantation $(P=.01)$. The best forced expiratory volumes in 1 second of control patients were comparable with those in surfactant lungs 4 weeks after transplant.

Conclusions: This study indicates a protective effect of exogenous surfactant instillation to donor lungs before retrieval on post-lung transplantation surfactant function and on early clinical outcome. This approach may help to improve the outcome after lung transplantation in the future.

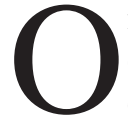
ne of the major obstacles in clinical lung transplantation is the occurrence of reperfusion injury, which may lead to life-threatening primary graft dysfunction. The importance of this issue initiated an enormous effort in research related to experimental lung transplantation. The majority of investigations focused on the improvement of pulmonary preservation. With the introduction of 


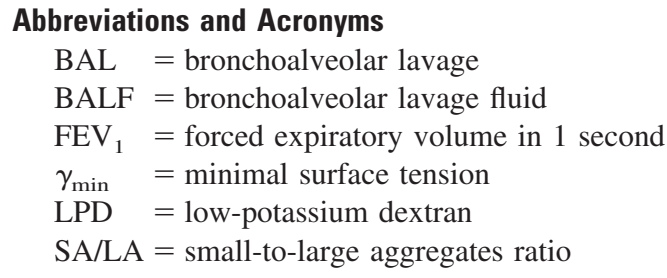

Euro-Collins flush perfusion, a worldwide standard was announced. However, although ischemic times were limited to 6 hours only, the initial graft function remained unreliable and unpredictable. One of the findings was impairment of surfactant function after reperfusion, a unique feature in pulmonary preservation and transplantation. In experimental studies, substitution of exogenous surfactant was used to prevent primary graft dysfunction.

We were able to demonstrate a beneficial effect of surfactant substitution on graft function in recipients with severe experimental reperfusion injury. ${ }^{1}$ A quantum leap in lung preservation was achieved with the introduction of low-potassium dextran (LPD) solution for flush preservation. A significant improvement of postoperative graft function was reported in clinical ${ }^{2,3}$ and experimental studies, and better surfactant function was shown. ${ }^{4}$ The purpose of this study was twofold: The first question addressed was whether the improvements of surfactant function resulting from LPD solution could be verified in clinical lung transplantation. The second aim was to define whether substitution of exogenous surfactant to the donor at the time of lung retrieval has an impact on surfactant function beyond the reperfusion period. The latter question was to be answered by laboratory assessment of surfactant composition and function and not by the early clinical course. Owing to the high variability of lung recipients and the high costs of exogenous surfactant, such a study would have been seriously underpowered and not feasible.

\section{Patients and Methods}

In a prospective, monocenter, randomized, investigator-initiated trial, 30 donor lungs for elective double lung transplantation were assessed by standard criteria before retrieval. All donors were evaluated consecutively and those with severe neurologic pulmonary edema were excluded from this study. On acceptance of the graft, randomization was performed and a bronchoalveolar lavage (BAL) was obtained from the right middle lobe. In the control group $(n=14)$, lungs were flushed antegradely via the pulmonary artery with LPD solution (4-6 L) at $8^{\circ} \mathrm{C}$ within 10 minutes while mechanical ventilation was continued. Lungs were excised en bloc and stored at a mild inflated state in cold $\left(4^{\circ} \mathrm{C}\right)$ preservation solution. In the surfactant group $(\mathrm{n}=15)$, a bovine surfactant preparation (Alveofact; Thomae, Germany) was instilled bronchoscopically (100 mg/kg body weight of the donor) and distributed in aliquots of 2 to $3 \mathrm{~mL}$ to all segments of both lungs. Thereafter,
TABLE 1. Patient characteristics

\begin{tabular}{lcc}
\hline & \multicolumn{2}{c}{ Recipients } \\
\cline { 2 - 3 } & Surfactant & Control \\
\hline Height (cm) & $169 \pm 7.2$ & $169 \pm 9.6$ \\
Age (y) & $34 \pm 11$ & $41 \pm 13$ \\
Female/male & $9 / 6$ & $8 / 6$ \\
Total lung capacity (L) & $5.6 \pm 0.9$ & $5.7 \pm 0.8$ \\
Diagnosis & & \\
$\quad$ Emphysema & 5 & 4 \\
Cystic fibrosis & 5 & 5 \\
Fibrosis & 2 & 2 \\
Other & 3 & 3 \\
\hline
\end{tabular}

preservation and harvesting were conducted as described for the control group. One double lung graft was not transplanted and was excluded from the study for severe infiltrations found ex situ in both lower lobes. Bilateral sequential lung transplantation was carried out in all recipients via anterolateral minithoracotomies as we described previously. ${ }^{5}$ In 12 recipients, the transplant procedure was carried out with the support of cardiopulmonary bypass. Use of cardiopulmonary bypass was statistically comparable in the surfactant and control groups $(P=.91)$. After transplantation, recipients were transferred to the intensive care unit and their lungs were mechanically ventilated in a pressure-controlled mode. BAL was obtained 18 to 24 hours after graft reperfusion.

\section{Donor Characteristics}

The main causes of deaths in the group of organ donors were subarachnoidal bleeding (31\%) and head injury (28\%). The mean donor age was $34.6 \pm 11.2$ years (range, 14-53 years). There were 17 male and 12 female donors, which were mechanically ventilated for $77 \pm 65$ hours (15-240 hours) before multiorgan donation. The ischemia time ranged from 210 to 640 minutes with a mean of $392 \pm 42$ minutes. There was no significant difference in these demographics between the surfactant and the control groups. Gender was not statistically different between the surfactant group ( 8 female donors of 15) compared with the control group (6 female donors of 14).

\section{Recipient Characteristics}

Recipient age, gender, and diagnosis are shown in Table 1. All patients were candidates for elective pulmonary transplantation. None was receiving mechanical or noninvasive ventilatory support before transplant. Informed consent for the study was obtained from each recipient. This trial was approved by the local ethics committee of the Hannover Medical School. Patient insurance for the trial was supplied.

The immunosuppressive protocol in all study and historical control patients consisted of a triple drug regimen of cyclosporine A (trough levels $250-350 \mathrm{ng} / \mathrm{mL}$ for the first 6 months after lung transplantation and 200-300 ng/mL thereafter), mycophenolic acid (2-3 g/day according to body weight), and prednisone (starting with $1 \mathrm{mg} / \mathrm{kg}$ body weight, followed by a taper regimen to a target dose of $0.1 \mathrm{mg} / \mathrm{kg}$ body weight over 3 weeks). No immunosuppressive induction therapy was administered. Acute rejection was 
diagnosed clinically; surveillance biopsy specimens were not taken. No clinical signs of rejection were found in any patients 10 days before or after pulmonary function testing.

\section{Surfactant Preparation}

The bovine surfactant preparation (Alveofact) was supplied by a generous donation from Thomae, Germany. The dry preparations were dissolved according to the manufacturer's instructions at the donor site immediately before bronchoscopic instillation.

\section{Surfactant Analysis}

Bronchoalveolar lavage fluid (BALF) was obtained from the right middle lobe in all donors before lung preservation with $100 \mathrm{~mL}$ of normal saline solution. The lavage was transported with the graft cooled at $4^{\circ} \mathrm{C}$. On arrival at the transplant center, BALFs were immediately centrifuged at $150 \mathrm{~g}$ and the cell-free supernatant was kept frozen at $-80^{\circ} \mathrm{C}$ until further analysis. A second BAL from of the left lingula lobe was taken after 18 to 24 hours of graft reperfusion, centrifuged, prepared, and then kept frozen as above.

Aliquots of the cell-free supernatant were assayed for total protein content by standard technique. For surfactant analysis, BAL surfactant lipids were separated into a large surfactant aggregate-containing pellet and a small surfactant aggregatecontaining supernatant by centrifugation of the cell-free BALF at $48,000 \mathrm{~g}$ for 60 minutes. Phospholipids in the small aggregatecontaining supernatant and in the large-aggregate pellet were determined by phosphorus analysis and their weight expressed as a small-to-large aggregate ratio (SA/LA). The surfactant pellet was resuspended in normal saline supplemented with $1.5 \mathrm{mmol} / \mathrm{L}$ calcium chloride to a phospholipid concentration of $1 \mathrm{mg} / \mathrm{mL}$. Surfactant function was determined with a pulsating bubble surfactometer (Electronetics, Buffalo, NY) according to the technique described by Enhorning. ${ }^{6}$ In brief, $40 \mu \mathrm{L}$ of the adjusted largeaggregate suspension was filled into the sample chamber. The surface tension at minimal bubble size $\left(\gamma_{\min }\right)$ was obtained after 5 minutes of bubble pulsation at a rate of 20 cycles/min and a temperature of $37^{\circ} \mathrm{C}$. Before bubble pulsation was started, the initial surface tension after bubble formation was measured and the adsorption rate was determined as surface tension 10 seconds after formation of a bubble. All analog data were digitalized and recorded by a personal computer.

\section{Lung Function Analysis}

Spirometry analysis (forced expiratory volume in 1 second $\left[\mathrm{FEV}_{1}\right]$ and forced vital capacity) was performed 4 weeks after lung transplantation. Results in the surfactant group were compared with those of controls as well as with 154 consecutive double lung transplant recipients outside this study (historic control). In addition, the best $\mathrm{FEV}_{1}$ was determined within the first year after lung transplantation and compared between the two control groups and the surfactant group.

\section{Statistical Analysis}

Data are expressed as mean \pm standard deviation. For data without repeated measurement, analysis of variance was applied. All data were analyzed with the Statistical Program of Social Sciences (SPSS for MS Windows Version 12.0; SPSS, Inc, Chicago Ill).

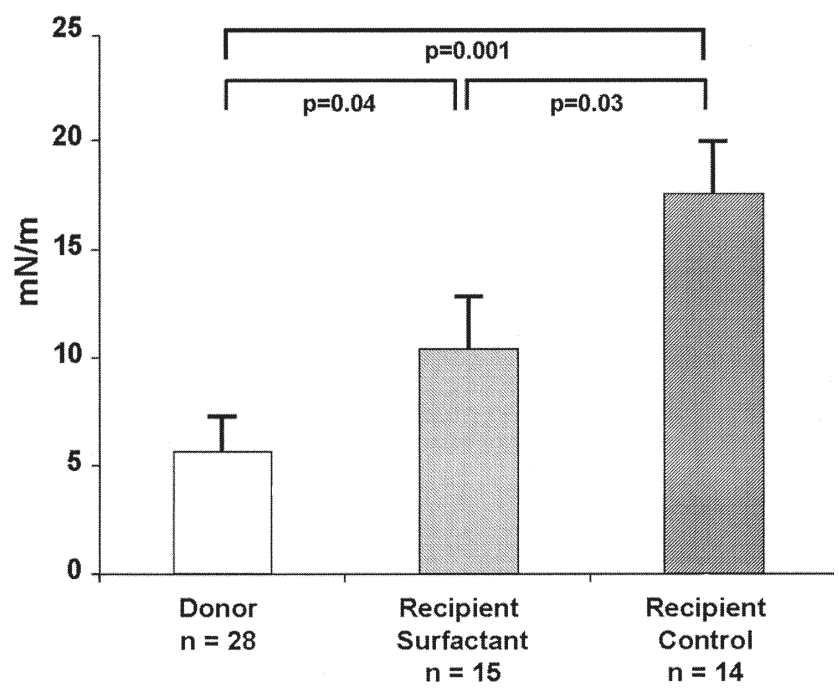

Figure 1. Minimal surface tension $\left(\gamma_{\min }\right)$ in donor lungs before retrieval. BALF, Bronchoalveolar lavage fluid.

\section{Results}

All recipients survived the follow-up period of 1 year. There was no difference in statistical relevance with respect to ischemic time (second transplanted lung), time of ventilatory support after transplantation, and length of stay in the intensive care unit or in the hospital between the two groups. Oxygenation index and lung compliance representing initial graft function were also comparable. Employing the arterial oxygenation/fraction of inspired oxygen ratio to evaluate early graft function 6, 12 and 24 hours after lung transplantation, these ratios in the nonsurfactant and surfactant populations were $327 \pm 87$ versus $322 \pm 105(P=$ $.89), 405 \pm 142$ versus $384 \pm 127(P=.71)$, and $430 \pm$ 160 versus $386 \pm 104(P=.42)$, respectively. Dynamic lung compliances in nonsurfactant and surfactant patients at 6,12 , and 24 hours after lung transplantation were $38 \pm 8$ versus $45 \pm 6 \mathrm{~mL} / \mathrm{mm} \mathrm{Hg}(P=.07), 40 \pm 11$ versus $44 \pm$ $11 \mathrm{~mL} / \mathrm{mm} \mathrm{Hg}(P=.43)$, and $42 \pm 11$ versus $49 \pm 12$ $\mathrm{mL} / \mathrm{mm} \mathrm{Hg}(P=.43)$, respectively.

Diagnosis of acute rejection led to methylprednisolone pulse therapy (1000 mg/day for 3 days) in 9 patients within the first 3 months after transplantation. In 5 patients $(n=2$, control group; $\mathrm{n}=3$, surfactant group), early treatment within 16 days after transplant was carried out; the remaining patients ( 2 of each group) received pulse therapy after the first month.

Surfactant function in the early postoperative period after lung transplantation was assessed by determination of minimal surface tension $\left(\gamma_{\min }\right)$ of BLF in the graft before harvesting and within 24 hours of transplantation. In all donor lungs a normal $\gamma_{\min }$ was found (Figure 1) before lung preservation. After transplantation, a significant loss of sur- 


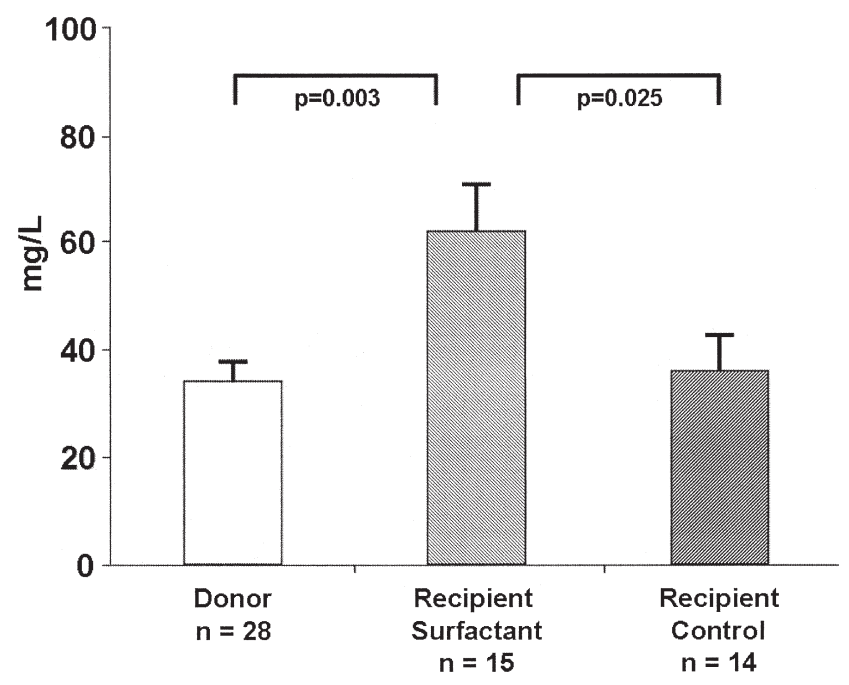

Figure 2. Phospholipids content of bronchoalveolar lavage fluids (BALF).

factant activity with an increase of $\gamma_{\text {min }}$ was observed. Although surfactant function in the control group was markedly impaired $\left(\gamma_{\min } 17.5 \pm 1.9 \mathrm{mN} / \mathrm{m}\right)$, instillation of surfactant before lung retrieval prevented the loss of surfactant function during the reperfusion period $\left(\gamma_{\min } 9.6 \pm 2.1\right.$ $\mathrm{mN} / \mathrm{m} ; P=.03$ compared with control). However, baseline of $\gamma_{\min }$ was not reached. Phospholipids were shown to be comparable before lung preservation and also after transplantation, as depicted in Figure 2. However, the surfactant instillation group showed higher phospholipid contents of the BAL after transplantation. The SA/LA ratio, which was already elevated before lung retrieval (all donors, $0.82 \pm 0.20$;

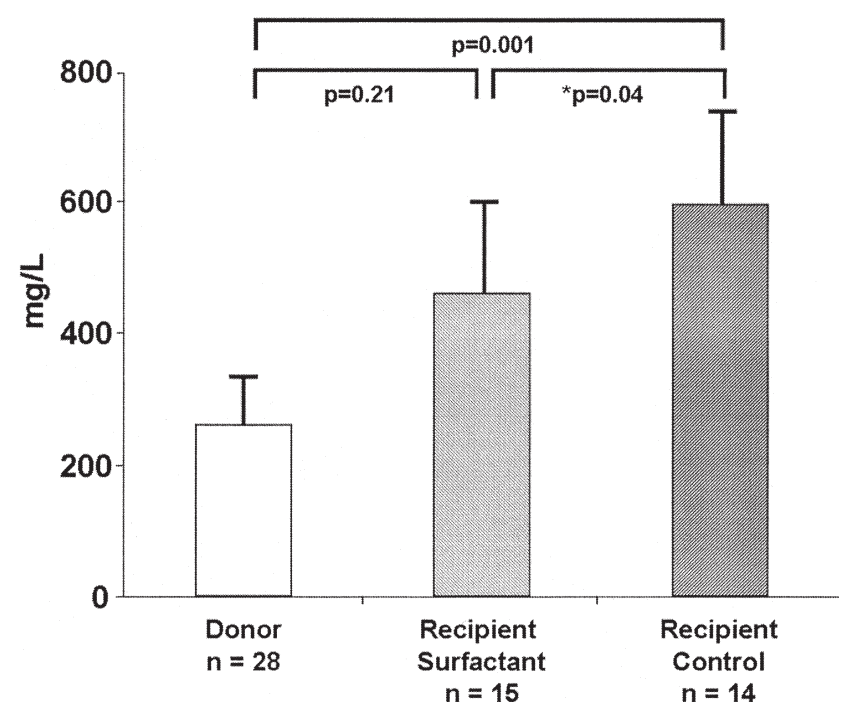

Figure 3. Protein content of bronchoalveolar lavage fluids (BALF). control group, $0.78 \pm 0.31$; surfactant group, $0.86 \pm 0.24$ ), was further increased in the control group (1.58 \pm 0.44$)$, indicating an increased metabolic conversion of surfactant into inactive small aggregates. In contrast, surfactant supplementation improved the $\mathrm{SA} / \mathrm{LA}$ ratio $(0.42 \pm 0.11 ; P=$ .01 compared with control), which indicates improved surfactant function.

Compared with measurements in previously reported healthy control subjects, ${ }^{7}$ BALF protein content was found slightly elevated before lung preservation in donor lungs in this study. In the control group (Figure 3), protein levels significantly increased from $234 \pm 72 \mathrm{mg} / \mathrm{L}$ before preservation to $598 \pm 141 \mathrm{mg} / \mathrm{L}$ after transplantation $(P=.04)$. In grafts that received surfactant instillation, posttransplant protein levels $(461 \pm 141 \mathrm{mg} / \mathrm{L})$ were not significantly higher than before preservation $(286 \pm 94 \mathrm{mg} / \mathrm{L} ; P=.21)$. Between both recipient groups (Figure 3), the difference of BAL protein content was also statistically significant.

Pulmonary function tests using spirometry 4 weeks after transplantation (Figure 4) showed a higher $\mathrm{FEV}_{1}$ in surfactant recipients compared with control patients. $\mathrm{FEV}_{1}$ values in the control group were comparable with those in a historic control cohort of 154 subsequent lung transplant patients. In both groups an $\mathrm{FEV}_{1}$ of $62 \% \pm 8 \%$ of the predicted value was reached. In contrast, the surfactant substitution group revealed a significantly higher $\mathrm{FEV}_{1}$ of $83 \% \pm 5 \%(P=.034$ and .001 , respectively $)$. During the first year after transplantation, pulmonary function testing was compared among all three groups using the best $\mathrm{FEV}_{1}$ as the main value. In the control group of this study, as well

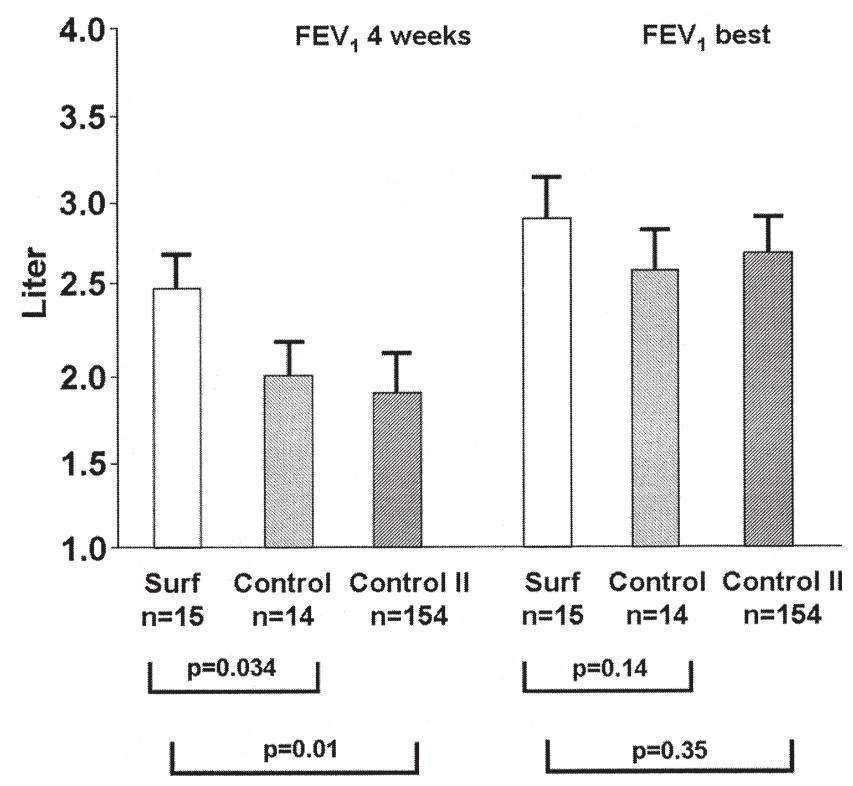

Figure 4. Forced expiratory volume in 1 second $\left(F E V_{1}\right) 4$ weeks after lung transplantation and best FEV1 in group comparison. Surf, Surfactant. 
as in the historical control group, a significant improvement compared with the measurement after 4 weeks was found $(P=.01)$ to a best $\mathrm{FEV}_{1}$ of $82 \% \pm 7 \%$. In the surfactant substitution group, a tendency of further improvement was noted. However, the best $\mathrm{FEV}_{1}$ of $90 \% \pm 6 \%$ of the predicted value was not significantly different from 4 -week posttransplant examinations of this group or the best $\mathrm{FEV}_{1}$ in control groups.

\section{Discussion}

Initial graft failure after lung transplantation is associated with surfactant dysfunction in the graft during the reperfusion period. ${ }^{8}$ It was established that preservation strategies using high potassium-containing solutions (eg, Euro-Collins solution) contributed to this phenomenon by two mechanisms: First, a direct toxic effect of these solutions leading to an impairment of the integrity and function of alveolar type II cells has previously been demonstrated. ${ }^{9}$ A second mechanism was seen in the increase of permeability of the alveolocapillary membrane to allow for influx of plasma proteins into the alveolar space, ${ }^{10}$ leading to an inhibition of surfactant function. ${ }^{11}$ These effects leading to impairment of graft function could be prevented by substitution of surfactant preparation before reperfusion of the graft in Euro-Collins-preserved grafts. ${ }^{8}$ Strategies were investigated when and how to substitute exogenous surfactant. ${ }^{12}$ However, with the introduction of LPD solutions in experimental studies, a protection of type II cells, as well as an improvement of surfactant function owing to protection of the integrity of the alveolocapillary membrane, was shown.

To our knowledge, this is the first clinical study investigating surfactant function in the donor lung before lung transplantation. There are two potential mechanisms by which impairment of surfactant function in donor organs before preservation could be induced. One mechanism is the development of pulmonary edema owing to brain death, so-called neurogenic pulmonary edema with subsequent surfactant inactivation by invading plasma proteins. A second mechanism could be ventilator-induced lung injury. It has been demonstrated that mechanical ventilation of normal lungs can induce an impairment of the pulmonary surfactant system. ${ }^{13}$ In our study, a normal biophysical surfactant function, an increased SA/LA ratio, and slightly elevated protein content was found in donor lungs before preservation. The elevated BALF protein indicates at least some leakage of plasma proteins into the alveolar space. It cannot be depicted whether this is more to the result of mechanical ventilation or neurogenic pulmonary edema. Interestingly, we found an increased SA/LA ratio, which might indicate that mechanical ventilation of donor lungs affects surfactant conversion. Importantly, we did not find a significant loss of the overall biophysical surfactant function, which suggests that both brain death and mechanical ventilation have little impact on the surfactant activity of donor lungs.

After lung transplantation, a decrease of surfactant function was found in the not substituted transplant recipients. This finding is in contrast to the experimental studies, which showed proper surfactant activity in the reperfusion period after lung transplantation. ${ }^{14}$ Indeed, the degree of impairment is comparable with that of patients receiving EuroCollins-preserved grafts in an earlier study. ${ }^{15} \mathrm{~A}$ further conclusion that can be drawn is that surfactant substitution to the graft before harvesting can be detected in the BALF 24 hours after transplantation by laboratory means to determine surface tension, protein content, and phospholipid levels. We were unable to show any differences in the postoperative course of the patients in group comparison. The impact of surfactant instillation on postoperative lung function was not strong enough to be detected in the small sample size of this study, and it has to be underscored that this study was not powered to detect such a difference. Initial graft function and early patient outcome are influenced by many factors, so a much larger cohort of patients is required to define the impact of surfactant substitution on the postoperative course. However, there was a strong association with lung function testing 4 weeks after transplantation and surfactant instillation. In untreated patients, as well as in a larger cohort of transplant recipients with LPD-preserved grafts (historical control), the $\mathrm{FEV}_{1}$ was only about $63 \%$ of the predicted value, and it took some weeks to months until the best $\mathrm{FEV}_{1}$ was reached. Therefore, it is likely that surfactant substitution has an impact on the recovery of donor lungs after transplantation, as suggested by the observed earlier recovery of $\mathrm{FEV}_{1}$ values. It can be speculated that surfactant substitution to the donor lung may indeed have an effect on lung recovery beyond the initial postoperative period.

Because preservation of the lung was not satisfactory in the past, numerous experimental as well as clinical studies were carried out to improve initial graft function. ${ }^{14}$ These efforts led to the introduction of LPD solution in clinical transplantation. ${ }^{2,3}$ After superior graft function was found also in observational clinical studies, LPD received worldwide acceptance as the new standard of lung preservation. As a sequel to this development, a remarkable increase of international lung transplant activities was observed, since more donor organs seemed to be acceptable and even socalled "marginal" grafts were successfully transplanted. However, this study indicates that there is still room for improvement of pulmonary preservation. It has been reported most recently ${ }^{16}$ that initial graft failure is still a major contributor to perioperative mortality after lung transplantation, with an incidence of about $20 \%$.

Our data show an increase of protein levels in BALF after transplantation, indicating the occurrence of a capillary 
leak and the development of pulmonary edema. Although this increase is moderate, it must be noted that the grafts used in this study were considered "acceptable" but not "marginal," with absence of significant pulmonary edema before preservation. It is speculative, but most likely, that the inhibition of the surfactant system is aggravated in so-called "marginal" grafts, which may already have some degree of capillary leakage before ischemia and reperfusion, possibly related to donor brain death and mechanical ventilation.

Further improvements to lung preservation can be achieved by modifying preservation solutions. We were able to show previously that the addition of iloprost, ${ }^{17}$ glutathione, ${ }^{18}$ or glycine ${ }^{19}$ to the flush solution improved initial graft function after extended hypothermic ischemia in a pig model of unilateral lung transplantation. Another approach is the application of retrograde pulmonary perfusion techniques to achieve a more homogenous protection of the donor lung. ${ }^{20}$

In conclusion, this study indicates a protective effect of surfactant substitution to the lung graft before preservation. In the past, repeated application of surfactant before preservation, before implantation, and during reperfusion was suggested. In our experience, instillation at a singular time point seems to be sufficient. It must be noted that implantation of surfactant-treated grafts was technically more demanding because deflation of the graft during implantation does not occur to the usual extent. The use of surfactant preparation in clinical lung transplantation was rare in the past owing to the high costs. However, the amount of surfactant preparation used in this study is quite low compared with the use in adult respiratory distress syndrome. ${ }^{21}$ Cost-effectiveness of surfactant substitution should be discussed at least for "marginal" grafts to further increase the acceptance rate of those donor lungs. It may also help to prevent initial graft dysfunction after lung transplantation.

\section{References}

1. Warnecke G, Struber M, Fraud S, Hohlfeld JM, Haverich A. Combined exogenous surfactant and inhaled nitric oxide therapy for lung ischemia-reperfusion injury in minipigs. Transplantation. 2001;71: 1238-44.

2. Struber M, Wilhelmi M, Harringer W, Niedermeyer J, Anssar M, Kunsebeck A, et al. Flush perfusion with low potassium dextran solution improves early graft function in clinical lung transplantation. Eur J Cardiothorac Surg. 2001;19:190-4.

3. Fischer S, Matte-Martyn AJ, De Perrot M, Waddell TK, Sekine Y, Hutcheon M, et al. Low-potassium dextran preservation solution improves lung function after human lung transplantation. $J$ Thorac Cardiovasc Surg. 2001;121:594-6.
4. Struber M. What is the role of surfactant and inhaled nitric oxide in lung transplantation? Crit Care. 2002;6:186-7. Epub 2002 May 9.

5. Fischer S, Struber M, Simon AR, Anssar M, Wilhelmi M, Leyh RG, et al. Video-assisted minimally invasive approach in clinical bilateral lung transplantation. J Thorac Cardiovasc Surg. 2001;122:1196-8.

6. Enhorning G. Pulsating bubble technique for evaluating pulmonary surfactant. J Appl Physiol. 1977;43:198-203.

7. Hohlfeld JM, Ahlf K, Enhorning G, Balke K, Erpenbeck VJ, Petschallies J, et al. Dysfunction of pulmonary surfactant in asthmatics after segmental allergen challenge. Am J Respir Crit Care Med. 1999;159: 1803-9.

8. Novick RJ, Gilpin AA, Gehman KE, Ali IS, Veldhuizen RA, Duplan $\mathrm{J}$, et al. Mitigation of injury in canine lung grafts by exogenous surfactant therapy. J Thorac Cardiovasc Surg. 1997;113:342-53.

9. Maccherini M, Keshavjee SH, Slutsky AS, Patterson GA, Edelson JD. The effect of low-potassium-dextran versus Euro-Collins solution for preservation of isolated type II pneumocytes. Transplantation. 1991; 52:621-6.

10. Veldhuizen RA, Lee J, Sandler D, Hull W, Whitsett JA, Lewis J, et al. Alterations in pulmonary surfactant composition and activity after experimental lung transplantation. Am Rev Respir Dis. 1993;148: $208-15$.

11. Seeger W, Stohr G, Wolf HR, Neuhof H. Alteration of surfactant function due to protein leakage: special interaction with fibrin monomer. J Appl Physiol. 1985;58:326-38.

12. Novick RJ, MacDonald J, Veldhuizen RA, Wan F, Duplan J, Denning $\mathrm{L}$, et al. Evaluation of surfactant treatment strategies after prolonged graft storage in lung transplantation. Am J Respir Crit Care Med. 1996;154:98-104.

13. Veldhuizen RA, Marcou J, Yao LJ, McCaig L, Ito Y, Lewis JF. Alveolar surfactant aggregate conversion in ventilated normal and injured rabbits. Am J Physiol. 1996;270(1 Pt 1):L152-8.

14. Struber M, Hohlfeld JM, Fraund S, Kim P, Warnecke G, Haverich A. Low-potassium dextran solution ameliorates reperfusion injury of the lung and protects surfactant function. J Thorac Cardiovasc Surg. 2000;120:566-72.

15. Hohlfeld JM, Tiryaki E, Hamm H, Hoymann HG, Krug N, Haverich A, et al. Pulmonary surfactant activity is impaired in lung transplant recipients. Am J Respir Crit Care Med. 1998;158:706-12.

16. Meyers BF, de la Morena M, Sweet SC, Trulock EP, Guthrie TJ, Mendeloff EN, et al. Primary graft dysfunction and other selected complications of lung transplantation: a single-center experience of 983 patients. J Thorac Cardiovasc Surg. 2005;129:1421-9.

17. Gohrbandt B, Sommer SP, Fischer S, Hohlfeld JM, Warnecke G, Haverich A, et al. Iloprost to improve surfactant function in porcine pulmonary grafts stored for twenty-four hours in low-potassium dextran solution. J Thorac Cardiovasc Surg. 2005;129:80-6.

18. Sommer SP, Gohrbandt B, Fischer S, Hohlfeld JM, Warnecke G, Avsar M, et al. Glutathione improves the function of porcine pulmonary grafts stored for twenty-four hours in low-potassium dextran solution. J Thorac Cardiovasc Surg. 2005;130:864-9.

19. Gohrbandt B, Fischer S, Warnecke G, Avsar M, Sommer SP, Haverich A, et al. Glycine intravenous donor preconditioning is superior to glycine supplementation to low-potassium dextran flush preservation and improves graft function in a large animal lung transplantation model after 24 hours of cold ischemia. J Thorac Cardiovasc Surg. 2006;131:724-9.

20. Struber M, Hohlfeld JM, Kofidis T, Warnecke G, Niedermeyer J, Sommer SP, et al. Surfactant function in lung transplantation after 24 hours of ischemia: advantage of retrograde flush perfusion for preservation. J Thorac Cardiovasc Surg. 2002;123:98-103. Comment in: J Thorac Cardiovasc Surg. 2002;123:3-5.

21. Gregory TJ, Steinberg KP, Spragg R, Gadek JE, Hyers TM, Longmore WJ, et al. Bovine surfactant therapy for patients with acute respiratory distress syndrome. Am J Respir Crit Care Med. 1997;155:1309-15. 